Cox, N. C. and Hill, Andrew P. ORCID: https://orcid.org/0000-0001-6370-8901 (2018) Trait perfectionism and attitudes towards people with disabilities. Personality and Individual Differences, 122. pp. 184-189.

Downloaded from: http://ray.yorksj.ac.uk/id/eprint/2612/

The version presented here may differ from the published version or version of record. If you intend to cite from the work you are advised to consult the publisher's version: https://www.sciencedirect.com/science/article/pii/S0191886917306335

Research at York St John (RaY) is an institutional repository. It supports the principles of open access by making the research outputs of the University available in digital form. Copyright of the items stored in RaY reside with the authors and/or other copyright owners. Users may access full text items free of charge, and may download a copy for private study or non-commercial research. For further reuse terms, see licence terms governing individual outputs. Institutional Repository Policy Statement

\title{
RaY
}

Research at the University of York St John

For more information please contact RaY at ray@yorksj.ac.uk 
Cox, N. C., \& Hill, A. P. (in press). Trait perfectionism and attitudes towards people with disabilities. Personality and Individual Differences. Accepted for publication 21/10/2017

Trait perfectionism and attitudes towards people with disabilities

Nathan C. Cox and Andrew P. Hill

York St John University, UK

Running head: Perfectionism and disability

Word Count: 4881

DECLARATION OF INTEREST: None

Contact details: Andrew P. Hill, Ph. D., School of Sport, York St. John University, Lord Mayor's Walk, York, YO31 7EX, UK, E-mail: a.hill@yorksj.ac.uk 


\begin{abstract}
Attitudes towards people with disabilities play an integral role in determining social inclusion. Unfortunately, however, attitudes are often negative and based on views of disability that are focused on impairment. The current study aimed to examine whether a commitment to perfection and flawlessness, in the form of trait perfectionism, predicted attitudes towards people with disabilities. A cross-sectional survey-based design was used. One hundred and eighty-eight university students completed measures of trait perfectionism (self-oriented, socially prescribed, and other-oriented) and an indirect measure of attitudes towards people with disabilities (negative affect, interpersonal stress, calm, positive cognitions and distancing behavior). A series of multiple regression analyses revealed that socially prescribed perfectionism positively predicted negative affect, interpersonal stress, and distancing behavior. The other two trait dimensions of perfectionism did not predict any aspect of attitudes towards people with disabilities. This findings can be explained by the relationship between socially prescribed perfectionism and difficulties interacting with others generally or, alternatively, the projection of socially prescribed beliefs on to others when measuring attitudes in an indirect fashion (i.e., other people are perceived to have negative attitudes towards those with disability).
\end{abstract}




\section{Trait perfectionism and attitudes towards people with disabilities}

The World Report for Disabilities estimates that 1 in 7 people have a disability worldwide (World Health Organisation; WHO). Despite how common disabilities are, people with disabilities are often subject to negative attitudes that promote prejudice, infringe on their rights and independence, and contribute to greater social exclusion (Barnes \& Mercer, 2001; Vilchinsky \& Findler, 2004). For example, people with disabilities face additional challenges when accessing higher education (Watson et al., 2017), employment (Nota, Santilli, Ginevra, \& Soresi, 2014), and healthcare (Tervo \& Palmer, 2004). People with disabilities are also aware that they are disadvantaged by others and often cite others' negative attitudes as one of the impediments to leading a full and purposeful life (e.g., Richardson, Smith, \& Papthomas, 2016). With these issues in mind, it is important to investigate factors which influence formation of attitudes towards people with disabilities. The current study aims to do so by examining whether a commitment to perfection and flawlessness, in the form of trait perfectionism, predicts attitudes towards people with disabilities.

\section{Attitudes towards people with disabilities}

Attitudes are typically considered to be an evaluation, favourable or unfavorable, of an object, person or concept (Fazio \& Petty, 2008). Attitudes have three main components: affective, cognitive, and behavioural (Zanna \& Rempel, 1988). As described by others in this area (Vilchinsky, Werner, \& Vilchinsky, 2010), the affective component of an attitude refers to the positive or negative emotions evoked by an object, person, or concept. The cognitive component refers to an individual's thoughts, perceptions, beliefs, opinions, and mental conceptualisations of an object, person or concept. Finally, the behavioural component refers to the way in which an individual intends to, or does, act towards an object, person or concept. Attitudes differ in 
valence, or direction, reflecting positive, negative, or neutral evaluations (Hewstone, Stroebe, \& Jonas, 2012). Attitudes also differ in strength, expressed by the degree of certainty or uncertainty of an individual's evaluation (Hewstone et al., 2012).

Attitudes towards people with disabilities will depend to a large degree on of the manner in which disability is construed. Historically, among the general public disability has been viewed in a negative manner, with heavy emphasis on impairment (Goodley, 2013). However, there have been some suggestions that views have begun to change with an increasing emphasis on disabling barriers imposed by society (Oliver, 2013). In terms of how these views might influence attitudes, a positive attitude may form when individuals believe people with disabilities can participate fully in society, whereas a negative attitude may form when individuals believe people with disabilities place a burden on society (Morin, Rivard, Crocker, Boursier, \& Caron, 2013). In accord, individuals report a range of different attitudes towards people with disabilities. On one hand, responses can include pity or fear, as well as hostility (Findler, Vilchinsky, \& Werner, 2007). On the other hand, responses can be more relaxed and positive (Findler et al., 2007).

One model that has been used to study attitudes towards disability is provided by Findler, Werner, and Vilchinsky (2007). Findler et al. (2007; Vilchinsky, Werner, \& Findler, 2010) use the three components of attitude (affective, cognitive, behavioural) as the basis to measure five specific dimensions of attitude. Three of the five dimensions relate to the affective component, negative affect (a person's most negative feelings), interpersonal stress (state of high emotional stress), and calm (a person's positive and relaxed emotional state). A further dimension relates to the cognitive component, positive cognitions (the positive valence of thoughts). The final dimension relates to the behavioral component, distancing behavior (passive or escapism 
behaviours). This approach uses an explicit assessment of attitudes requiring people to consider and reflect upon theirs and others views in a conscious manner (Wilson \& Scior, 2015). It also uses an indirect assessment of attitudes in that personal attitudes are measured via perceptions of how others respond to encounters with people with disabilities. In this manner, respondents project their own attitudes into the situation. This feature is considered to be a particular strength when measuring attitudes towards disability as it can help avoid response distortions (e.g., socially desirable responses) (Antonak \& Livneh, 2000).

There is a large body of research examining attitudes towards people with disabilities. Previous research has focused on the influence of demographic factors, including gender (Vilchinsky, Werner, \& Findler, 2010) culture (Benomir, Nicolson, \& Beail, 2016) and personality (Keller \& Siegrist, 2010). An individual's familiarity and contact with people with disabilities in the form of their profession has also been examined (e.g., healthcare and business; Rosenthal, Chan, \& Livneh, 2006; Chan, Lee, Yuen, \& Chan, 2002), along with the type of disability, including physical (Vilchinsky, Findler, \& Werner, 2010) and intellectual (Benomir et al., 2016) disabilities. This research has found, for example, that more positive attitudes are typically held by females, by those with more knowledge and contact with people with disabilities, by individuals lower in neuroticism and higher in openness, and towards physical, rather than intellectual disabilities. As evidenced by these findings, the formation of attitudes towards people with disabilities is complex and influenced by a range of factors, including individual differences.

\section{Multidimensional perfectionism}

Perfectionism is a multidimensional personality characteristic that can be studied using a range of different models. Within one popular model, Hewitt and Flett (Hewit \& Flett, 2004, 
2006) define perfectionism as the perceived requirement, or actual requirement, to be perfect.

They emphasize the importance of three trait dimensions of perfectionism: self-oriented perfectionism (SOP; perfectionistic standards imposed on self), other-oriented perfectionism (OOP; perfectionistic standards imposed on others), and socially prescribed perfectionism (SPP; the perception that others impose perfectionistic standards). This model is popular as it provides a means of studying both intrapersonal (i.e., SOP) and interpersonal dimensions of perfectionism (i.e., SPP and OOP). It is also popular as it is part of a much broader model that includes other aspects of perfectionism such as perfectionistic self-presentation styles and perfectionistic cognitions (Hewitt, Flett, \& Mikail, 2017).

Research has found SOP, SPP, and OPP to be related to unique outcomes. SOP is a complex dimension of perfectionism. On one hand, it is related to seemingly desirable achievement behaviours but, on the other hand, it is also related to less desirable features such as self-criticism and contingent self-worth that provide the basis for psychological difficulties (Flett \& Hewitt, 2006). By contrast, SPP is more clearly related to negative outcomes. Perhaps most strikingly, SPP is consistently related to clinical outcomes such as depression and suicide ideation (Kiamanesh, Dyregov, Haavind, \& Dieserud, 2014). Finally, unlike the two other dimensions, OOP is predominately related to interpersonal outcomes. Again, the outcomes can include some seemingly desirable behaviors such as assertiveness. However, it is also related to dominance, narcissism and aggression making it especially problematic in interpersonal contexts (Stoeber, 2014, 2015).

\section{Perfectionism and attitudes towards people with disabilities}

As personality characteristics have been found to influence attributes toward disability, it is possible that perfectionism will also do so. Intuitively, as OOP is the most interpersonal 
dimension of perfectionism it may be the most important in terms of attitudes towards people with disabilities. OOP encapsulates highly critical other-directed tendencies, including imposing the need for perfection on others. These tendencies and apparent disregard for the feelings of others implies little interest in the circumstances or welfare of people with disabilities. There is indirect empirical evidence to support this possibility. For example, Stoeber (2015) found OOP to be related to the dark triad traits of narcissism, Machiavellianism, and psychopathy. These are traits that are related to intense self-interest, exploitive behaviours, and a notable disregard for others. In addition, Stoeber $(2014,2015)$ found OOP to be related to lower levels of social goals that include nurturance (i.e., making other people feel happy), altruism, prosocial values, and interest in others, as well as higher callous and uncaring traits.

As SPP reflects beliefs regarding pressures from significant others, including society more widely (the "generalised other"), this dimension of perfectionism is likely to be the next most important dimension of perfectionism regarding attitudes towards disability. SPP includes important distorted beliefs about unrealistic societal expectations. If these views are projected on to others, SPP may be related to negative attitudes towards people with disabilities when measured in an indirect fashion (i.e., it may promote perceptions that people are generally unaccepting of people with disabilities and that people with disabilities are also subject to pressure to be perfect). SPP may also be related to negative attitudes towards people with disabilities due to general difficulties in social interactions. An irrational need for approval and fear of negative evaluation, for example, make social interactions stressful and this may extend to interacting with people with disabilities. In support of this possibility, research has found that SPP is related to perceptions of poorer relations with others (Flett, Hewitt, Shapiro, \& Rayman, 
2001), higher anticipation of negative interactions with others (Nepon, Flett, Hewitt, \& Monar, 2011) and higher social anxiety (Cox \& Chen, 2015).

As the most intrapersonal dimension perfectionism, SOP may be the least important with regards to attitudes toward people with disabilities. In addition, in contrast to both OOP and SPP, it is also possible SOP may be related to positive attitudes towards people with disabilities. This is because inclusive to the notion of high personal standards might also be self-expectations regarding how one should behave towards others (Stoeber, 2015). That is, because societal attitudes towards disability have slowly shifted to being more positive, SOP may present more socially desirable attitudes towards those with disability (i.e., a more positive attitude is the "right" attitude to have). Current evidence is supportive of this possibility in that research has not typically found SOP to be related to either the social anxiety that characterises SPP or the lack of concern for others that characterise OOP (Nepon, Flett, Hewitt, \& Molnar, 2011; Stoeber, 2014, 2015). Rather, SOP has been found to be positively related to social goals that include nurturance and altruism, and negatively related to callous and uncaring traits (Stoeber, 2014, 2015).

\section{The current study}

The aim of the current study is to examine whether perfectionism predicts attitudes towards people with disabilities. It was hypothesised that (H1) OOP would predict negative attitudes towards people with disabilities (higher negative affect, interpersonal stress, and distancing behaviour with lower calm and positive cognitions). It was also hypothesized that (H2) SPP would predict negative attitudes towards people with disabilities (higher negative affect, interpersonal stress, and distancing behaviours with lower calm and positive cognitions). Finally, it is hypothesised that (H3) SOP would predict positive attitudes towards people with 
disabilities (higher positive cognitions and calm with lower negative affect, interpersonal stress and distancing behaviour).

\section{Methods}

\section{Participants}

Participants were 188 university students (age $M=20.84, S D 2.81$, range 18-33, males $=58$, females $=130$ ) who were recruited using convenience sampling at the university to which the authors are affiliated. Students reported that they were enrolled on various degree programmes, the largest being psychology $(n=41)$, occupational therapy $(n=30)$, physiotherapy $(n=22)$, English $(n=17)$, and primary education $(n=13)$. Most participants were white $(n=$ 177), single $(n=159)$, and in either their first $(n=69)$ or third $(n=83)$ academic year of their degree programme. All participants were volunteers. No incentives were offered for taking part. Participants completed a paper-and-pencil questionnaire or an electronic version (sent directly to them) which was then returned at the participants' convenience. Ethical approval was granted prior to beginning the study.

\section{Instruments}

Perfectionism. Perfectionism was measured using a shortened version of Hewitt and Flett's (1991) Multidimensional Perfectionism Scale (MPS; Cox, Enns, \& Clara, 2002). The MPS comprises three subscales measuring SOP (5-items e.g., 'One of my goals is to be perfect in everything I do'), OOP (5-reverse coded items, e.g., 'I feel people are far too demanding of me') and SPP (5-items, e.g., 'I do not have high standards for those around me'). Participants were asked to rate each item on a 7-point scale from 1 (strongly disagree) to 7 (strongly agree). Evidence has been provided by Cox et al., (2002) to support the reliability and validity of the instrument. 
Attitudes Towards People with Disabilities. Attitudes towards people with disabilities were measured using the shortened version of the Multidimensional Attitudes Scale Towards Persons with Disabilities (MAS; Findler, et al., 2007; Vilchinsky et al., 2010). The MAS asks participants to respond to a vignette which describes a casual social interaction between either 'Joseph' or 'Michelle' and a person using a wheelchair. Participants are then asked how Joseph or Michelle would react to the scenario. The MAS comprises 22-items and five subscales, negative affect assessing a person's most negative feelings (5-items, e.g., 'Upset'), interpersonal stress assessing stress-related feelings (4-items, e.g., 'Tension'), calm assessing positive relaxed feelings (3-items, e.g., 'Serenity'), positive cognitions assessing favorable thoughts about the interaction (5-items, e.g., 'He/she looks friendly'), and distancing behavior assessing behaviors indicative of passivity or desire to avoid the interaction (5-items, e.g., 'Find an excuse to leave'). Participants were sex matched, so males reacted to the Joseph vignette and the person in the wheelchair was male. Females reacted to the Michelle vignette and the person in the wheelchair was female. This was done to remove any cross-gender effects in attitude found in previous research (Vilchinsky, Werner, \& Findler, 2010) Evidence has been provided by Findler et al., (2007) and Vilchinsky, Werner, and Findler (2010) of support the reliability and validity of the instrument.

\section{Results}

\section{Preliminary analysis}

Data analysis was conducted using IBM SPSS Statistics version 23. One participant was removed from the data set as a male completed the female version of the questionnaire. Then variables were screened for univariate and multivariate outliers using procedures outlined by Tabachnick and Fidell (2007). One univariate outlier (z-score $>3.29, p<.001$, two tailed) and 
one multivariate outlier (Mahalanobis distance $>\chi^{2}[13]=26.31, \mathrm{p}<.001$ ) were removed. Most variables remained significantly skewed to varying degrees. Therefore, subsequent bivariate correlations and multiple regression analyses, including standard errors and hypothesis tests, are based on $95 \%$ bias-corrected accelerated (BCa) bootstrap estimates (1000 resamples). Internal reliabilities (Cronbach's alpha) were assessed for the scales and were acceptable (Table 1).

Descriptive and bivariate correlations

Descriptive statistics following the removal of outliers are reported in Table 1. For trait perfectionism variables, SPP and OOP scores were low-to-moderate (3 to 4 on a seven-point scale) whereas SOP scores were moderate-to-high (5 to 6 on a seven-point scale). Most attitudinal variables were low-to-moderate ( 2 to 3 on a five-point scale). A comparison of the perfectionism scores to normative nonclinical population scores for the relevant age range provided by Hewitt and Flett (2004) revealed that the current participants reported slightly higher than average SOP $(\mathrm{T}$-Score $=50$ versus 56 for males and 56 females $)$, slight lower than average SPP $(\mathrm{T}-$-Score $=50$ versus 45 males and 44 females $)$ and near average OOP scores $(\mathrm{T}$-Score $=50$ versus 50 males and 51 females). ${ }^{i}$

Bivariate correlations between dimensions of perfectionism and attitudes towards people with disabilities are shown in Table 1. SPP showed small sized positive correlations with negative affect and distancing behaviours ( $r=.10$; Cohen, 1992). Also, SPP showed a medium sized positive correlation with interpersonal stress $(r=.30$; Cohen, 1992). OOP had a small sized negative correlation with negative affect ( $r=.10$; Cohen, 1992). SOP did not significantly correlate with any attitudinal variables. Regression of trait perfectionism influences on attitudes towards people with disabilities ${ }^{i i}$ 
A series of regression analyses were used to assess the predictive ability of the three traits of perfectionism on attitudes towards people with disabilities. The results of the analyses are reported in Table 2 .

Negative affect. In combination, the three perfectionism dimensions explained a significant amount of the variance in negative affect. SPP uniquely predicted negative affect, showing a small positive significant relationship. SOP and OOP did not significantly predict negative affect.

Interpersonal stress. In combination, the three perfectionism dimensions explained a significant amount of the variance in interpersonal stress. SPP uniquely predicted interpersonal stress, showing a medium positive relationship. SOP and OOP did not significantly predict interpersonal stress.

Distancing behaviour. In combination, the three perfectionism dimensions explained a significant amount of the variance in distancing behaviour. SPP uniquely predicted distancing behaviours, showing a small positive significant relationship. SOP and OOP did not significantly predict distancing behavior.

Calm and positive cognitions. In combination, the three dimensions of perfectionism did not explain a significant amount of the variance in calm or positive cognitions. In both cases, none of the dimensions were unique predictors.

\section{Discussion}

The aim of the current study was to examine whether perfectionism predicted attitudes towards people with disabilities. It was hypothesised that (H1) OOP and (H2) SPP would predict negative attitudes (higher negative affect, interpersonal stress, and distancing behaviour with lower calm and positive cognitions), whereas (H3) SOP would predict positive attitudes (higher 
positive cognitions and calm with lower negative affect, interpersonal stress and distancing behaviour).

\section{Multidimensional perfectionism and attitudes towards people with disabilities}

In support of the hypotheses, SPP positively predicted negative affect, interpersonal stress, and distancing behaviour dimensions of attitudes towards disabilities. As the relationship was limited to these particular three dimensions, and the largest relationship was for interpersonal stress, the findings appear consistent with the notion that social stress interacting with others, generally, and likely perceived difficulty interacting with those with disability, in particular, may explain this finding. Anxiety and nervousness associated with not knowing how to interact with people with disabilities is common (Morgan \& Lo, 2013). Higher levels of SPP may carry increased susceptibility to this type of negative emotional response. Moreover, given that interactions with people with disabilities may be anticipated as stressful and evoke negative feelings, the distancing behavior related to SPP is understandable. Distancing behavior is typical of the general coping strategies associated with SPP in other contexts (e.g., Chen, Hewitt, Flett, Cassels, Birch, \& Blasberg, 2012) and offers a means of avoiding interactions with people with disabilities.

One alternative explanation for the findings regarding socially prescribed perfectionism pertains to the indirect manner in which attitudes were measured in the current study. Although indirect measurement avoids some of the response difficulties associated with direct measurement and has been advocated in this area (Antonak \& Livneh, 2000), the type of indirect measurement used in the MAS means that personal attitudes may not actually be measured but, rather, perceptions of others' attitudes. As such it is possible that the current findings reflect perceptions that others are less accepting of people with disabilities. If this is the case, rather 
than conclude that SPP is related to more negative attitudes towards people with disabilities, it would be more accurate to conclude that SPP is related to perceptions that others have more negative attitudes towards people with disabilities. At the moment, whether the findings reflect personal attitudes or the projection of socially prescribed beliefs cannot be ascertained. Future research is required using direct measures of attitude towards people with disabilities. Regardless, this study is the first to provide evidence that trait perfectionism is related to attitudes towards people with disabilities (or, at least, is related to perceptions of other peoples' attitudes towards people with disabilities).

Contrary to the hypotheses, OOP did not significantly predict any of the dimensions of attitude. This is difficult to reconcile with the findings elsewhere regarding the callous and uncaring nature of OOP (Stoeber, 2014, 2015). There are a number of possible explanations for this finding. Perhaps other-directed expectations are limited to individuals (e.g., spouse) and groups (e.g., workmates) who are considered important and do not extend to strangers regardless of whether they have a disability or not. Alternatively, perhaps other-directed expectations do extend to other people but the general callous and uncaring behaviors do not extend to those with disabilities. Individuals with disability may be spared demanding expectations because it might be considered unreasonable to hold them to the same standards as others or such people are disregarded completely (i.e., not considered worthy of attention in any way). Again, it is also possible that due to the indirect measurement of attitudes, OOP may be related to certain personal attitudes but say little about what one might anticipate other people's attitudes to be. If this is the case, whether OOP is related to attitudes towards people with disabilities is still unknown. 
Like OOP, SOP did not significantly predict any of the dimensions of attitude. SOP is regarded as an intrapersonal dimension of perfectionism as it is primarily concerned with personal thoughts, feelings, and behaviours rather than those of others. In this regards, the findings are less surprising than those that pertain to OOP. However, recent research suggests that SOP is related to more prosocial attitudes and goals (Stoeber, 2014, 2015) hence our expectation that SOP may predict more positive attitudes. On the basis of the two studies prior to this one, Stoeber (2015) speculated that SOP may promote more care and kindness to others. There was no evidence of this in context of disability in the current study. As was the case for OOP, our findings regarding SOP and attitudes towards people with disabilities are therefore inconclusive. As such, and in light of findings elsewhere, additional research directed at whether $\mathrm{SOP}$ is related to greater care for others, negative feelings towards others, or disinterest is required.

In exploring the relationship between perfectionism and attitudes towards disability further, we envisage that different perfectionism variables may be useful (see Flett \& Hewitt, 2016). For example, perfectionistic self-presentation (PSP; i.e. the need to appear perfect to other people and not display or disclose imperfections in public; Hewitt et al., 2003) may be important in regards to how individuals perceive they should or should not behave when interacting with people with disabilities and how they feel about these interactions. As such, PSP may explain additional variance in attitudes towards people with disabilities or even be a mediating factor by encapsulating general unease in social interactions. The positive relationship between PSP and public self-consciousness, fear of negative evaluation, and social anxiety support this possibility (Hewitt et al., 2003). We therefore consider the development and test of a "social unease" model 
of the relationship between perfectionism and attitudes towards people with disabilities (and other minority groups) to be an important avenue for future research.

\section{Limitations and other future directions}

There were a number of notable limitations in the current study. The study adopted a cross-sectional design. This means no causal relationships can be inferred. Longitudinal work is an important next step in this regard. The study also adopted a particular approach to measuring perfectionism and attitudes to disability in a specific group (i.e., wheelchair users). Caution is required not to generalize findings beyond these features. For example, instruments designed to measure perfectionism vary in content and may have different relationship with attitudes. The attitude measure we used was explicit, indirect, and included a scenario featuring physical disability. It may be that findings change when using attitudes measures that are implicit, direct and focused on other specific disabilities or types of disability (e.g., intellectual). The study also used self-reported responses to a hypothetical scenario which should not be conflated with actual behavior that may differ. The sample was drawn from a student population, here with a majority of females from health and social care/sciences. This group may well display more positive attitudes towards disability so findings should not be assumed to generalize to other populations. With this in mind, and in hope of improving the lives of people with disabilities, we would particularly like to see future research examine the current relationships among individuals who have contact with people with disabilities as part of their professions (e.g., doctors, nurses, and physiotherapists).

\section{Conclusion}

The current study found SPP to predict more negative attitudes towards people with disabilities. This finding may be because of general stress when interacting with others or the 
projection of socially prescribed beliefs when measuring attitudes in an indirect manner (i.e., other people are perceived to have negative attitudes towards those with disability). 


\section{References}

Antonak, R. F., \& Livneh, H. (2000). Measurement of attitudes towards persons with disabilities. Disability and rehabilitation, 22(5), 211-224.

Barnes, C., \& Mercer, G. (2001). Disability culture. Handbook of disability studies, 515-534.

Benomir, A. M., Nicolson, R. I., \& Beail, N. (2016). Attitudes towards people with intellectual disability in the UK and Libya: a cross-cultural comparison. Research in developmental disabilities, 51, 1-9.

Chan, C. C., Lee, T., Yuen, H. K., \& Chan, F. (2002). Attitudes towards people with disabilities between Chinese rehabilitation and business students: An implication for practice. Rehabilitation Psychology, 47(3), 324-338.

Chen, C., Hewitt, P. L., Flett, G. L., Cassels, T. G., Birch, S., \& Blasberg, J. S. (2012). Insecure attachment, perfectionistic self-presentation, and social disconnection in adolescents. Personality and Individual Differences, 52(8), 936-941.

Cohen, J. (1992). A power primer. Psychological bulletin, 112, 155-159.

Cox, B. J., Enns, M.W., \& Clara, I. P. (2002). The multidimensional structure of perfectionism in clinically distressed and college student samples. Psychological Assessment, 14, 365-373.

Cox, S. L., \& Chen, J. (2015). Perfectionism: A contributor to social anxiety and its cognitive processes. Australian Journal of Psychology, 67(4), 231-240.

Fazio, R. H., \& Petty, R. E. (Eds.). (2008). Attitudes: key readings: their structure, function, and consequences. Hove: Psychology Press.

Findler, L., Vilchinsky, N., \& Werner, S. (2007). The Multidimensional Attitudes Scale toward Persons with Disabilities (MAS): Construction and validation. Rehabilitation Counseling Bulletin, 50(3), 166-176. 
Flett, G. L., \& Hewitt, P. L. (2006). Positive versus negative perfectionism in psychopathology: A comment on Slade and Owens's dual process model. Behavior modification, 30(4), 472-495.

Flett, G. L., \& Hewitt, P. L. (2016). Still measuring perfectionism after all these years: Reflections and an introduction to the special issue on advances in the assessment of perfectionism. Journal of Psychoeducational Assessment, 34(7), 615-619.

Flett, G. L., Hewitt, P. L., Shapiro, B., \& Rayman, J. (2001). Perfectionism, beliefs, and adjustment in dating relationships. Current Psychology, 20(4), 289-311.

Goodley, D. (2013). Dis/entangling critical disability studies. Disability \& Society, 28(5), 631644.

Hewitt, P. L., \& Flett, G. L. (1991). Perfectionism in the self and social contexts: Conceptualization, assessment, and association with psychopathology. Journal of personality and social psychology, 60(3), 456-470.

Hewitt, P. L., \& Flett, G. L. (2004). Multidimensional perfectionism scale (MPS): Technical manual. MHS.

Hewitt, P. L., Flett, G. L., \& Mikail, S. F. (2017). Perfectionism: a relational approach to conceptualization, assessment, and treatment. Guilford Publications.

Hewitt, P., Flett, G., Sherry, S., Habke,M., Parkin,M., Lam, R., et al. (2003). The interpersonal expression of perfection: Perfectionistic self-presentation and psychological distress. Journal of Personality and Social Psychology, 84, 1303-1325.

Hewstone, M., Stroebe, W., \& Jonas, K. (2012). An introduction to social psychology. Chichester: John Wiley \& Sons. 
Hill, A. P., Robson, S. J., \& Stamp, G. M. (2015). The predictive ability of perfectionistic traits and self-presentational styles in relation to exercise dependence. Personality and Individual Differences, 86, 176-183.

Keller, C., \& Siegrist, M. (2010). Psychological resources and attitudes toward people with physical disabilities. Journal of Applied Social Psychology, 40(2), 389-401.

Kiamanesh, P., Dyregrov, K., Haavind, H., \& Dieserud, G. (2014). Suicide and perfectionism: A psychological autopsy study of non-clinical suicides. OMEGA-Journal of Death and Dying, 69(4), 381-399.

Morgan, P. E., \& Lo, K. (2013). Enhancing positive attitudes towards disability: evaluation of an integrated physiotherapy program. Disability and rehabilitation, 35(4), 300-305.

Morin, D., Rivard, M., Crocker, A. G., Boursier, C. P., \& Caron, J. (2013). Public attitudes towards intellectual disability: a multidimensional perspective. Journal of Intellectual Disability Research, 57(3), 279-292.

Nepon, T., Flett, G. L., Hewitt, P. L., \& Molnar, D. S. (2011). Perfectionism, negative social feedback, and interpersonal rumination in depression and social anxiety. Canadian Journal of Behavioural Science/Revue canadienne des sciences du comportement, 43(4), 297-308.

Nota, L., Santilli, S., Ginevra, M. C., \& Soresi, S. (2014). Employer attitudes towards the work inclusion of people with disabilities. Journal of Applied Research in Intellectual Disabilities, 27(6), 511-520.

Oliver, M. (2013). The social model of disability: thirty years on. Disability \& society, 28(7), 1024-1026. 
Richardson, E. V., Smith, B., \& Papathomas, A. (2017). Disability and the gym: experiences, barriers and facilitators of gym use for individuals with physical disabilities. Disability and rehabilitation, 39(19), 1950-1957.

Rosenthal, D. A., Chan, F., \& Livneh, H. (2006). Rehabilitation students' attitudes toward persons with disabilities in high-and low-stakes social contexts: A conjoint analysis. Disability and rehabilitation, 28(24), 1517-1527.

Stoeber, J. (2014). How other-oriented perfectionism differs from self-oriented and socially prescribed perfectionism. Journal of Psychopathology and Behavioral Assessment, 36(2), 329-338.

Stoeber, J. (2015). How other-oriented perfectionism differs from self-oriented and socially prescribed perfectionism: Further findings. Journal of Psychopathology and Behavioral Assessment, 37(4), 611-623.

Tabachnick, B. G., \& Fidell, L. S. (2007). Using multivariate statistics (5th ed.). Boston: Allyn and Bacon.

Tervo, R. C., \& Palmer, G. (2004). Health professional student attitudes towards people with disabilities. Clinical rehabilitation, 18(8), 908-915.

Vilchinsky, N., \& Findler, L. (2004). Attitudes Toward Israel's Equal Rights for People With Disabilities Law: A Multiperspective Approach. Rehabilitation psychology, 49(4), 309316.

Vilchinsky, N., Findler, L., \& Werner, S. (2010). Attitudes toward people with disabilities: The perspective of attachment theory. Rehabilitation Psychology, 55(3), 298-306. 
Vilchinsky, N., Werner, S., \& Findler, L. (2010). Gender and attitudes toward people using wheelchairs: A multidimensional perspective. Rehabilitation Counseling Bulletin, 53(3), 163-174.

Watson, A.C., Fulambarker, A., Kondrat, D.C., Holley, L.C., Kranke, D., Wilkins, B.T., Stromwall, L.K. and Eack, S.M., (2017). Social work faculty and mental illness stigma. Journal of Social Work Education, 53(2), 174-186.

Wilson, M. C., \& Scior, K. (2015). Implicit attitudes towards people with intellectual disabilities: their relationship with explicit attitudes, social distance, emotions and contact. PloS one, 10(9).

World Health Organization. (2011). World report on disability. World Health Organization.

Zanna, M. P., \& Rempel, J. K. (1988). Attitudes: A new look at an old concept. In D. Bar-Tal \& A. W. Kruglanski (Eds.), The social psychology of knowledge (pp. 315-334). Cambridge, UK: University Press. 
Table 1 Descriptive statistics and bivariate correlations of perfectionism and attitudes towards people with disabilities.

\begin{tabular}{|c|c|c|c|c|c|c|c|c|c|c|}
\hline Variable & $M$ & $S D$ & $\alpha$ & 1 & 2 & 3 & 4 & 5 & 6 & 7 \\
\hline 1. Self-oriented perf. & 5.08 & 1.13 & .85 & & & & & & & \\
\hline 2. Socially prescribed perf. & 3.28 & 1.19 & .80 & $.29 * *$ & & & & & & \\
\hline 3. Other-oriented perf. & 3.85 & 1.17 & .77 & $.17 *$ & $-.16^{*}$ & & & & & \\
\hline 4. Negative affect & 2.02 & 0.69 & .76 & .07 & $.23 * *$ & $-.16^{*}$ & & & & \\
\hline 5. Interpersonal stress & 2.90 & 0.89 & .74 & -.02 & $.32 * *$ & -.11 & $.54 * *$ & & & \\
\hline 6. Calm & 2.24 & 0.78 & .78 & .03 & -.17 & .07 & $-.23 * *$ & $-.29 * *$ & & \\
\hline 7. Positive cognitions & 3.64 & 0.66 & .84 & .01 & -.10 & -.01 & $-.17 *$ & -.11 & $.30 * *$ & \\
\hline 8. Distancing behaviour & 2.05 & 0.75 & .82 & .05 & $.20 * *$ & -.09 & $.40 * *$ & $.37 * *$ & $-.20 * *$ & $-.18^{*}$ \\
\hline
\end{tabular}


Table 2 Regression analyses of perfectionism and attitudes towards people with disabilities.

\begin{tabular}{|c|c|c|c|c|c|}
\hline Model & $\mathrm{B}$ & SE & $p$ & $\mathrm{BCa} 95 \% \mathrm{Cl}$ & $\beta$ \\
\hline \multicolumn{6}{|l|}{ Negative affect } \\
\hline \multicolumn{6}{|c|}{$F(3,178)=4.22, p<.01 ; R^{2}=.07$} \\
\hline Self-oriented perf. & .01 & .05 & .81 & {$[-.09, .11]$} & .02 \\
\hline Socially prescribed perf. & .12 & .05 & .02 & {$[.02, .22]$} & .21 \\
\hline Other-oriented perf. & -.07 & .04 & .13 & {$[-.16, .02]$} & -.12 \\
\hline \multicolumn{6}{|l|}{ Interpersonal stress } \\
\hline \multicolumn{6}{|c|}{$F(3,177)=7.51, p<.01 ; R^{2}=.11$} \\
\hline Self-oriented perf. & -.09 & .06 & .18 & {$[-.21, .04]$} & -.11 \\
\hline Socially prescribed perf. & .26 & .06 & .00 & {$[.15, .36]$} & .34 \\
\hline Other-oriented perf. & -.03 & .06 & .65 & {$[-.14, .09]$} & -.04 \\
\hline
\end{tabular}

Calm

$F(3,178)=2.15, p>.05 ; \mathrm{R}^{2}=.04$

Self-oriented perf.

$.05 \quad .06$

$.39 \quad[-.08, .18]$

.08

Socially prescribed perf.

$-.12$

.05

$.02[-.22,-.02]$

Other-oriented perf.

.02

.05

$.76 \quad[-.08, .11]$

.03

Positive cognitions

$F(3,178)=1.02, p>.05, \mathrm{R}^{2}=.02$

Self-oriented perf.

.04

.05

$.38 \quad[-.04, .13]$

.07

Socially prescribed perf.

$-.07$

.05

$.11 \quad[-.16, .02]$

$-.13$

Other-oriented perf.

$-.03$

.05

$.52 \quad[-.12, .08]$

$-.05$

Distancing behaviour 
$F(3,179)=2.78, p<.05 ; \mathrm{R}^{2}=.05$

Self-oriented perf.

Socially prescribed perf.

Other-oriented perf.
$-.01$

.13

$-.04$
.05

.05

.05
$.91 \quad[-.10, .09]$ $-.01$

Note. $* p<0.05, * * p<0.01$, two-tailed; Perf = Perfectionism; BCa =Bias-corrected accelerated. $\beta$ is not part of bootstrap estimates.

\section{Endnotes}

${ }^{\mathrm{i}}$ These norms are based on a 45-item version of the MPS. So to allow comparison with the norms using the 15-item version used in the current study, the mean item score was times by 15 (number of items per subscale for the longer version of the MPS).

${ }^{\text {ii }}$ We also completed a series of supplementary regression analyses that repeated the regressions reported here. These supplementary analyses included an additional predictor block consisting of gender (dummy coded as male $=0$ and female $=1$ ). The gender predictor block was not a significant predictor of negative affect, interpersonal stress, calm, and distancing behaviors $(p<.05$; variance explained was $1 \%$ to $2 \%)$. In these cases, there were also no substantive changes evident in the results of the second predictor block/step that included trait dimensions of perfectionism plus gender in comparison to regressions reported here. However, in the case of positive cognitions, the gender predictor block/step was significant, $F(1,180)=8.71, p<.01, R^{2}$ $=.05$. Females reported significantly higher positive cognitions than males $(\beta=.22, \mathrm{BCa} \mathrm{B}=.31$, $\mathrm{SE}=.09,95 \% \mathrm{CI}=.11$ to .49$)$. Unlike the regression reported here, the second predictor block/step was also significant $F(1,177)=2.98, p<.05, R^{2}=.06$. However, none of the trait 
dimensions of perfectionism were significant unique predictors of positive cognitions: SOP ( $\beta$

$=.09, \mathrm{BCa} \mathrm{B}=.05, \mathrm{SE}=.05,95 \% \mathrm{CI}=-.05$ to .15$), \mathrm{SPP}(\beta=-.13, \mathrm{BCa} \mathrm{B}=-.07, \mathrm{SE}=.05,95 \%$

$\mathrm{CI}=-.17$ to .03$)$, and $\mathrm{OOP}(\beta=-.01, \mathrm{BCa} \mathrm{B}=-.01, \mathrm{SE}=.05,95 \% \mathrm{CI}=.10$ to .07$)$. 